-Note-

\title{
Japanese and Korean Population Data on Three STR Polymorphic Markeres; D3S1358, vWA and FGA
}

\author{
Kanako Yoshida*1, Jong Yeol Kim*2, Kentaro Kasai*1 and Hajime Sato*1 \\ National Research Institute of police Science, 6-3-1, Kashiwanoha, Kashiwa, \\ Chiba 277-0882, Japan*1, \\ The Supreme Public Prosecutor's Office, Seoul 137-730, Korea*2
}

(Received 9 August 2000; 10 October 2000)

\begin{abstract}
Allele frequency distributions of the D3S1358, vWA and FGA loci were determined in the Japanese and the Korean populations using AmpFlSTR ${ }^{\mathrm{TM}}$ Blue triplex system.

All three loci in the Korean population and the vWA and FGA loci in the Japanese population met the Hardy-Weinberg equilibrium expectation, except the D3S1358 locus in the Japanese population $(p=0.01)$. However, the D3S1358 departure from the Hardy-Weinberg equilibrium was no longer significant when using the conventional chi-square test. Allele frequency distributions of the D3S1358 and vWA loci in the Japanese population were not significantly different from those of the Korean population. The allele distribution of the Japanese data was similar to other previously reported Japanese data.
\end{abstract}

Key words: D3S1358, vWA, FGA, Japanese, Korean

\section{Introduction}

The typing of short tandem repeat (STR) polymorphisms is routinely used for forensic identification and the multiplex PCR system with automated detection using fluorescent labeled primers has increased the throughput potential for STR typing ${ }^{1,2)}$.

Recently, several kits which contain fluorescent labeled STR primers are on the market. AmpFlSTR ${ }^{\mathrm{TM}}$ Blue kit (Applied Biosystems, CA) contains single color labeled fluorescent primer sets which can coamplify three tetranucleotide repeat polymorphic loci; D3S1358 ${ }^{3)}$, $\mathrm{vWA}^{4)}$ and $\mathrm{FGA}^{5)}$. This kit is one of the most evaluated multiplex STR kits for forensic identification purposes ${ }^{6}$.

The present study investigated allele frequ- ency distributions for the three STR loci in the Japanese and the Korean populations and comparisons of allele frequency distributions between the Japanese or Korean population and other populations.

\section{Materials and methods}

Bloodstain samples collected from 254 Japanese and 152 Korean individuals were used for this study. DNA samples were purified from the bloodstains using phenol-chloroform extraction $^{7}$. The extracted DNA samples were quantitated by spectrophotometric method.

The D3S1358, vWA and FGA loci were coamplified using AmpFISTR ${ }^{\mathrm{TM}}$ Blue kit (Applied Biosystems, CA), according to the manufacture's protocol ${ }^{8)}$. The amplification was performed using GeneAmp ${ }^{\mathrm{TM}}$ PCR System 9600 
thermocycler (Applied Biosystems, CA) with one nanogram of DNA sample as template DNA. ABI PRISM ${ }^{\mathrm{TM}} 377$ DNA sequencer (Applied Biosystems, CA) was used for the electrophoresis of the PCR products. The results were analyzed with GeneScan ${ }^{\mathrm{TM}}$ Software (version 2.1), and allele fragment sizes were calculated using GS 350 internal standard marker and AmpFISTR $^{\mathrm{TM}}$ Blue allelic ladder loaded on a same gel.

The allele frequency for each locus was calculated by gene counting method. Statistical tests for fit to Hardy-Weinberg equilibrium expectations, linkage equilibrium and comparison study of allele frequencies between popula- tion samples were performed using exact test with computer program GENEPOP (version 1.2) using Markov chain method ${ }^{9}$.

\section{Results and Discussion}

Table 1 show the allele frequency distributions for the three STR loci from 254 Japanese population and 152 Korean population samples. The observed and expected heterozygosities and the power of discrimination (PD) on each locus were similar in both population samples.

All three loci in the Korean population and the vWA and the FGA loci in the Japanese population data were in good agreement with Hardy-Weinberg equilibrium expectation,

Table 1 Observed allele frequencies and statistical parameters on three STR loci in the Japanese and Korean population samples.

\begin{tabular}{|c|c|c|c|c|c|c|}
\hline \multirow[b]{2}{*}{ Allele } & \multicolumn{2}{|c|}{ D3S1358 } & \multicolumn{2}{|c|}{ vWA } & \multicolumn{2}{|c|}{ FGA } \\
\hline & $\begin{array}{l}\text { Japanese } \\
(\mathrm{N}=254)\end{array}$ & $\begin{array}{l}\text { Korean } \\
(\mathrm{N}=152)\end{array}$ & $\begin{array}{l}\text { Japanese } \\
(\mathrm{N}=254)\end{array}$ & $\begin{array}{c}\text { Korean } \\
(\mathrm{N}=152)\end{array}$ & $\begin{array}{l}\text { Japanese } \\
(\mathrm{N}=254)\end{array}$ & $\begin{array}{c}\text { Korean } \\
(\mathrm{N}=152)\end{array}$ \\
\hline 12 & & 0.007 & & & & \\
\hline 13 & & 0.003 & & & & \\
\hline 14 & 0.034 & 0.066 & 0.179 & 0.174 & & \\
\hline 15 & 0.392 & 0.391 & 0.032 & 0.030 & & \\
\hline 16 & 0.296 & 0.253 & 0.191 & 0.201 & & \\
\hline 17 & 0.201 & 0.214 & 0.289 & 0.263 & 0.006 & 0.003 \\
\hline 18 & 0.069 & 0.066 & 0.248 & 0.224 & 0.022 & 0.016 \\
\hline 19 & 0.008 & & 0.051 & 0.095 & 0.049 & 0.063 \\
\hline 20 & & & 0.008 & 0.013 & 0.102 & 0.059 \\
\hline 21 & & & 0.002 & & 0.126 & 0.102 \\
\hline 21.2 & & & & & & 0.003 \\
\hline 22 & & & & & 0.212 & 0.182 \\
\hline 22.2 & & & & & 0.006 & 0.003 \\
\hline 23 & & & & & 0.225 & 0.227 \\
\hline 23.2 & & & & & 0.002 & 0.010 \\
\hline 24 & & & & & 0.108 & 0.220 \\
\hline 24.2 & & & & & & 0.003 \\
\hline 25 & & & & & 0.083 & 0.079 \\
\hline 25.2 & & & & & 0.002 & \\
\hline 26 & & & & & 0.049 & 0.020 \\
\hline 27 & & & & & 0.008 & 0.007 \\
\hline 28 & & & & & & 0.003 \\
\hline H obs. & 0.71 & 0.74 & 0.78 & 0.81 & 0.87 & 0.85 \\
\hline H exp. & 0.71 & 0.78 & 0.78 & 0.80 & 0.85 & 0.84 \\
\hline PD & 0.86 & 0.90 & 0.92 & 0.93 & 0.96 & 0.95 \\
\hline Exact test & 0.01 & 0.37 & 0.39 & 0.96 & 0.54 & 0.10 \\
\hline
\end{tabular}


except the D3S1358 locus in the Japanese population sample which deviated from HardyWeinberg equilibrium expectation $(p=0.01)$ using the exact test. However, using conventional chi-square test, the D3S1358 locus in the Japanese population sample show no evidence of deviation from Hardy-Weinberg equilibrium $(0.25<\mathrm{p}<0.1)$.

Allele frequency distributions were compared between the Japanese and the Korean population at the three STR loci. Allele frequency distributions of the D3S1358 and vWA loci in the Japanese population were not significantly different from those in the Korean population (D3S1358; $\mathrm{p}=0.08$, vWA; $\mathrm{p}=0.33$ ). The difference between Japanese and Korean populations on the FGA locus $(\mathrm{p}=0.0002)$ might be due to genetic difference or to small sample size.

Table 2 shows comparison studies of the Japanese and Korean population data with other population data reported previously. On all of the three loci, no significant differences were observed between our Japanese population data in this study and other Japanese population data reported previously. No significantly differences were observed between our Korean population data and other Asian population data except one Japanese population data in the FGA locus ( $p=$ 0.005).

In conclusion, Japanese and Korean population database of the three STR loci, D3S1358, vWA and FGA, were established and all three loci were revealed to be useful for forensic identity application for both Japanese and Korean population.

\section{Reference}

1) Buel, E., Schwartz, M. B. and LaFountain, M.J.: Capillary electrophoresis STR analysis: comparison to gel based systems. $J$. Forensic Sci., 43, 164-170 (1998).

2) Gill, P., Urquhart, A., Millican, E., Oldroyd, N., Watson, S., Sparkes, R. and Kimpton, C.P.: A new method of STR interpretation using inferential logic-development of a criminal intelligence database. Int. J. Leg. Med., 109, 14-22 (1996).

Table 2 Pairwise comparison of allele frequencies between the Japanese or the Korean population data and different population data.

\begin{tabular}{|c|c|c|c|}
\hline Japanese/Population & $\mathrm{p}$-value & Korean/Population & p-value \\
\hline \multicolumn{4}{|l|}{ D3S1358 } \\
\hline Japanese $^{10)}$ & 0.94 & Japanese $^{10)}$ & 0.15 \\
\hline Amazonian ${ }^{15)}$ & 0.17 & Amazonian ${ }^{15)}$ & 0.10 \\
\hline Brazilian in San Paulo ${ }^{15)}$ & 0.0003 & Brazilian in San Paulo' ${ }^{15)}$ & 0.15 \\
\hline \multicolumn{4}{|l|}{ vWA } \\
\hline Japanese $^{10)}$ & 0.1 & Japanese $^{10)}$ & 0.93 \\
\hline Japanese $\mathrm{e}^{11)}$ & 0.39 & Japanese $^{11)}$ & 0.88 \\
\hline Taiwanese ${ }^{12)}$ & 0.04 & Taiwanese ${ }^{12)}$ & 0.46 \\
\hline Amazonian ${ }^{15)}$ & 0.0001 & Amazonian ${ }^{15)}$ & 0.0003 \\
\hline Brazilian in San Paulo's) & 0.0000 & Brazilian in San Paulo ${ }^{15)}$ & 0.0002 \\
\hline \multicolumn{4}{|l|}{ FGA } \\
\hline Japanese $^{10)}$ & 0.26 & Japanese $^{10)}$ & 0.0005 \\
\hline Japanese $^{11)}$ & 0.37 & Japanese $^{11)}$ & 0.14 \\
\hline Chinese $^{13)}$ & 0.01 & Chinese $^{13)}$ & 0.25 \\
\hline Egyptian ${ }^{14)}$ & 0.03 & Egyptian ${ }^{14)}$ & 0.33 \\
\hline Yemenian ${ }^{14)}$ & 0.007 & Yemenian $^{14)}$ & 0.16 \\
\hline Amazonian ${ }^{15)}$ & 0.001 & Amazonian ${ }^{15)}$ & 0.003 \\
\hline Brazilian in San Paulo15) & 0.0000 & Brazilian in San Paulo15) & 0.005 \\
\hline
\end{tabular}


3) Li, H., Schmidt, L., Wei, M. H., Hustad, T., Lerman, M. I. Zbar, B. and Tory, K.: Three tetranucleotide polymorphisms for loci: D3S1352; D3S1358; D3S1359. Hum. Mol. Genet., 2, 1327 (1993).

4) Kimpton, C., Walton, A. and Gill, P.: A further tetranucleotide repeat polymorphism in the vWF gene. Hum. Mol.Genet., 1, 287 (1992).

5) Mills, K. A., Evan, D. and Murray, J. C.: Tetranucleotide repeat polymorphism at the human alpha fibrinogen locus (FGA). Hum. Mol. Genet., 1, 779 (1992).

6) Wallin, J.M., Buoncristiani, M.R., Lazaruk, K. D., Fildes, N., Holt, C. L. and Walsh, P. S.: TWGDAM validation of the AmpFlSTR $^{\text {TM }}$ Blue PCR amplification kit for forensic casework analysis. J. Forensic Sci., 43, 117-134 (1998).

7) Sambrook, J., Fritsch, E.F. and Maniatis, T. Molecular Cloning, A Laboratory Manual, 2nd edu. Cold Spring Harbor Laboratory Press, USA (1989).

8) AmpFlSTR ${ }^{\mathrm{TM}}$ Blue PCR Amplification Kit, User's Manual, P/N 402827, Perkin Elmer Corporation, Foster City, CA (1996).

9) Guo, S.W. and Thompson, E.A.: Performing the exact test of HardyWeinberg proportion for multiplex alleles. Biometrics, 48, 361-372 (1992).

10) Yamamoto, T., Uchihi, R., Nozawa, H., Huang, X. L., Leong, Y. K., Tanaka, M., Mizutani, M., Tamaki, K. and Katsumata,
Y.: Allele distribution at nine STR lociD3S1358, vWA, FGA, TH01, TPOX, CSF1PO, D5S818, D13S317 and D7S820- in the Japanese population by multiplex PCR and capillary electrophoresis. J. Forensic Sci., 44, 167-170 (1999).

11) Nagai, A., Yamada, S., Watanabe, Y., Bunai, Y. and Ohya, I.: Analysis of STR loci HUMF13A01, HUMFXIIIB, HUMLIPOL, HUMTH01, HUMTPOX and HUMVWFA31 in a Japanese population. Int. J. Leg. Med., 109, 34-36 (1996).

12) Lee, J. C. I.; Chen, C. H., Tsai, L. C., Linacre, A. and Chang, J. G.: The screening of 13 short tandem repeat loci in the Chinese population. Forens. Sci. Int., 87,137-144 (1997).

13) Rolf, B., Waterkamp, K. and Hühne, J.: Allele frequency data for the FGA locus in eight populations. Int. J. Leg. Med., 111, 55 -56 (1998).

14) Klintschar, M., Kozma, Z., Al Hammadi, N., Abdull Fatah, M. and Nöhammer, C.: A study on the short tandem repeat systems HumCD4, HumTH01 and HumFIBRA in population samples from Yemen and Egypt. Int. J. Leg. Med., 111, 107-109 (1998).

15) Corte-Real, F., Andrade, L., Anjos, M.J., Carvalho, M., Vieira, D. N., Carracedo, A. and Vide, M. C.: Population genetics of nine STR loci in two population from Brazil. $J$. Forensic Sci., 45, 432-435 (2000). 